\title{
Escaping residual albuminuria in hypertension: should we start eplerenone or reduce salt intake?
}

\author{
Martin H. de Borst ${ }^{1} \cdot$ Gozewijn D. Laverman $^{2} \cdot$ Gerjan Navis $^{1}$
}

Received: 16 December 2018 / Revised: 19 December 2018 / Accepted: 21 December 2018 / Published online: 23 January 2019

(c) The Japanese Society of Hypertension 2019

The presence of albuminuria in patients with hypertension indicates an increased risk of cardiovascular disease, progressive kidney disease, and premature mortality, and is considered an independent treatment target. Pharmacological blockade of the renin-angiotensin-aldosterone system (RAAS) using angiotensin-converting enzyme (ACE) inhibitors or angiotensin receptor blockers (ARBs) forms the cornerstone of anti-albuminuric treatment. However, the therapy response is incomplete in many patients, resulting in residual albuminuria [1]. Residual albuminuria has been associated with a higher risk of reaching end-stage renal disease and adverse cardiovascular outcomes. Therefore, adjunct treatment targeting residual albuminuria is often needed.

Volume overload is a common factor underlying resistance to RAAS blockade, and co-treatment with a thiazide diuretic has been shown to be effective in further reducing residual albuminuria in both diabetic [2] and non-diabetic [3] patients. A similar effect was shown for the mineralocorticoid receptor antagonist (MRA) eplerenone in the EVALUATE trial, which enrolled non-diabetic patients [4]. In addition to reducing volume overload via its diuretic effect, MRAs adjunct to an ACE-inhibitor or ARB treatment can provide additional reno- and cardio-protection through suppression of the so-called "aldosterone escape." This escape entails residual aldosterone production despite blockade of the angiotensin II type 1 receptor, as well as angiotensin II type 2 receptors, which promote cardiac and renal fibrosis. Recently, high aldosterone levels were shown

Martin H. de Borst

m.h.de.borst@umcg.nl

1 Division of Nephrology, Department of Internal Medicine, University of Groningen, University Medical Center Groningen, Groningen, The Netherlands

2 Department of Nephrology, Ziekenhuis Groep Twente (ZGT) Hospital, Almelo and Hengelo, The Netherlands to remain associated with worse renal function despite adequate RAAS blockade [5]. In addition, a higher serum potassium level elicited by ACE-inhibitor or ARB treatment may further stimulate aldosterone production. Therefore, MRA treatment is a relevant adjunct reno- and cardioprotective therapy, particularly in patients with residual albuminuria during optimized standard RAAS blockade.

Importantly, an effect size similar to that of diuretics treatment in terms of both albuminuria reduction and blood pressure reduction can be achieved by reducing dietary sodium intake [2, 3]. Sodium restriction and thiazide treatment have even been shown to provide additive albuminuria-lowering effects [2, 3]. In contrast, high dietary sodium intake abolishes the albuminuria-lowering effect of ACE-inhibitor or ARB treatment, and may also impede the renal and cardioprotective effects of this cornerstone treatment [6-8]. These observations underscore the risk of excessive sodium intake, particularly in patient groups susceptible to volume overload, including patients with chronic kidney disease or congestive heart failure [9].

To date, the combined impact of MRA treatment and sodium intake on residual albuminuria during standard RAAS blockade has not been studied. In this issue of Hypertension Research, Nishimoto et al. [10] addressed this topic in a post hoc analysis of the EVALUATE trial. Their hypothesis was that high salt intake activated MR independent of aldosterone levels; thus, MRA treatment during a high salt diet would reverse resistance to RAAS blockade in patients with hypertension and albuminuria. To investigate this hypothesis, the authors divided the participants in the EVALUATE trial, which previously demonstrated that adjunct treatment with eplerenone for 52 weeks further reduced albuminuria [4], into tertiles according to their baseline salt intakes. Salt intake was estimated using urinary sodium excretion in the first morning void, which is not the best method but is still an acceptable reflection of salt intake. More important than the method, the upper bound of the lowest tertile of salt intake was $189 \mathrm{mEq} /$ day, which equaled $4.3 \mathrm{~g} \mathrm{Na}^{+}$or $10.9 \mathrm{~g}$ of salt per day. This amount is 
already more than double the maximum salt intake recommended by the World Health Organization $(5 \mathrm{~g}$ of salt per day), indicating that even in the lowest tertile, the salt intake was on average relatively high, but was even higher for the other two tertiles. This observation is not surprising, given the known high salt intake in the Eastern Asian population, but is relevant to place this study into the context of other studies, which have been mainly performed in Europe [11]. The eplerenone-treated patients in the highest tertile of sodium excretion, in which patients consumed $>13.5 \mathrm{~g}$ of salt per day, displayed a significantly greater reduction in albuminuria than the placebo-treated individuals in the same tertile $(-22.5 \%$ vs. $+21.8 \%, p=$ $0.02)$. In contrast, patients in the lowest $(-10.2 \%$ vs. $-0.84 \%, p=0.65)$ or middle $(-19.5 \%$ vs. $+9.5 \%, p=$ 0.22 ) sodium tertile did not demonstrate an albuminurialowering effect of eplerenone. Similar systolic blood pressure changes were observed. The authors conclude that cotreatment with eplerenone may be effective in reducing residual albuminuria in patients with hypertension in the presence of what generally would be considered an excessively high sodium intake.

The work by Nishimoto et al. [10] positions eplerenone as another adjunct therapy that can be employed to manage patients with an incomplete response to RAAS blockade during (very) high sodium intake. Previous studies revealed that thiazide diuretics had a similar effect in reducing residual albuminuria. Interestingly, thiazides also seem to be effective in patients with a low sodium intake [2,3] (i.e., at sodium intake levels below the lowest tertile in the present study). Therefore, the interaction of MRA efficacy with sodium intake (i.e., during high but not intermediate or low sodium intake) in the study by Nishimoto could be considered slightly surprising, particularly, since at the group level, albuminuria was similar among the sodium tertiles at baseline. On the other hand, baseline albuminuria was in the microalbuminuria range on average in these patients $(\sim 100$ $\mathrm{mg} / \mathrm{g}$ ) and therefore there might not have been much further to win for the MRA. This finding is in contrast to those of prior studies with thiazides performed in macroalbuminuric patients allowing for a larger effect size.

The mechanism predominantly responsible for the adjunct albuminuria-lowering effect of eplerenone in this study remains uncertain. This mechanism could well be merely a diuretic effect, which would be in line with the thiazide diuretics discussed above. Unfortunately, the EVALUATE trial lacked a thiazide diuretic arm that would have allowed a direct comparison. A direct MR-mediated effect may also play a role. At first glance, this latter possibility may seem implausible, because a high salt intake will suppress rather than stimulate plasma aldosterone levels. In the current study, the baseline plasma

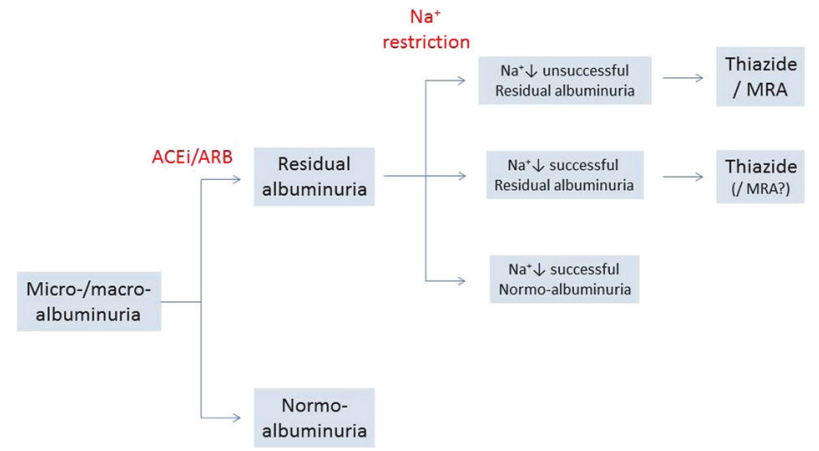

Fig. 1 Proposed algorithm for management of albuminuria in patients with hypertension. ACE-inhibitor or ARB treatment is considered the first-line therapy in conjunction with adequate (additional) blood pressure control. If residual albuminuria remains, patients should be advised to adhere to dietary sodium restriction. If unsuccessful, adjunct thiazide or MRA treatment may be initiated to further reduce albuminuria. When adequate salt restriction $(<2 \mathrm{~g}$ per day) is achieved as documented by $24 \mathrm{~h}$ sodium excretion but residual albuminuria remains, a thiazide diuretic may be prescribed

aldosterone levels were similar over the sodium intake tertiles. However, preclinical studies have implicated aldosterone-independent, MR-mediated, adverse effects of a high sodium intake, which may be mediated by the small GTPase Rac1 [12, 13]. This finding would implicate correction of aldosterone escape during ACE-inhibitor/ARB treatment by eplerenone as at least a partial explanation for the albuminuria-lowering effect.

The observation that MRA treatment is only effective in patients with a high sodium intake raises the question of where MRAs should be positioned in the treatment algorithm for albuminuria (a proposal is provided in Fig. 1). Given the proven efficacy, limited side effects, and low costs, we probably should invest more time and effort in reducing excessive sodium intakes. Globally, dietary sodium restriction is notoriously difficult to achieve, even in patients in whom a high salt intake may have important health implications, such as patients with chronic kidney disease [11]. A single recommendation by an individual, such as a physician or dietician, may not be sufficient to achieve sustained lowering of the salt intake. Adherence is particularly impeded by the widespread presence of salt in food products. To overcome this limitation, caregivers should discuss sodium intake structurally with their patients using current dietary information, preferably after obtaining 24-h urine samples. Integration of nutritional dashboards within medical patient records may facilitate data collection and translate the outcomes into information that is actionable for caregivers and patients. For example, nutritional information can be used to positively reinforce patients or to provide specific feedback. Moreover, the establishment of self-management programs [14] may help patients regain 
control of their health through extensive education, motivational interviewing, coaching, and self-monitoring of sodium intake, blood pressure, and albuminuria. Beyond the clinical setting, governments should take action to initiate stepwise regulation of salt content in food products. This process can be achieved through changes in food labeling, reformulation, and media campaigns, and probably by a combination of these approaches [15].

In patients who are unable to control their salt intake even after extensive efforts, adjunct treatment with a thiazide diuretic or a MRA may be the next step. The choice for either of the two approaches may depend on the individual patient setting, such as the presence of hyper- or hypokalemia. Patients with residual albuminuria despite adequate sodium restriction may be preferably treated with a thiazide diuretic, although more studies may be needed to definitively address whether adjunct MRA treatment is effective in this setting.

\section{Compliance with ethical standards}

Conflict of interest The authors declare that they have no conflict of interest.

Publisher's note: Springer Nature remains neutral with regard to jurisdictional claims in published maps and institutional affiliations.

\section{References}

1. Nehme A, Zibara K. Efficiency and specificity of RAAS inhibitors in cardiovascular diseases: how to achieve better end-organ protection? Hypertens Res. 2017;40:903-9.

2. Kwakernaak AJ, Krikken JA, Binnenmars SH, Visser FW, Hemmelder MH, Woittiez AJ, et al. Effects of sodium restriction and hydrochlorothiazide on RAAS blockade efficacy in diabetic nephropathy: a randomised clinical trial. Lancet Diabetes Endocrinol. 2014;2:385-95.

3. Vogt L, Waanders F, Boomsma F, de Zeeuw D, Navis G. Effects of dietary sodium and hydrochlorothiazide on the antiproteinuric efficacy of losartan. J Am Soc Nephrol. 2008;19:999-1007.
4. Ando K, Ohtsu H, Uchida S, Kaname S, Arakawa Y, Fujita T, et al. Anti-albuminuric effect of the aldosterone blocker eplerenone in non-diabetic hypertensive patients with albuminuria: a double-blind, randomised, placebo-controlled trial. Lancet Diabetes Endocrinol. 2014;2:944-53.

5. Gant CM, Laverman GD, Vogt L, Slagman MCJ, Heerspink HJL, Waanders F, et al. Renoprotective RAAS inhibition does not affect the association between worse renal function and higher plasma aldosterone levels. BMC Nephrol. 2017;18:370.

6. Heerspink HJL, Holtkamp FA, Parving HH, Navis GJ, Lewis JB, Ritz E, et al. Moderation of dietary sodium potentiates the renal and cardiovascular protective effects of angiotensin receptor blockers. Kidney Int. 2012;82:330-7.

7. Slagman MC, Waanders F, Hemmelder MH, Woittiez AJ, Janssen WM, Heerspink HJL, et al. Moderate dietary sodium restriction added to angiotensin converting enzyme inhibition compared with dual blockade in lowering proteinuria and blood pressure: randomised controlled trial. BMJ. 2011;343:d4366.

8. Vegter S, Perna A, Postma MJ, Navis G, Remuzzi G, Ruggenenti P. Sodium intake, ACE inhibition, and progression to ESRD. J Am Soc Nephrol. 2012;23:165-73.

9. Lambers Heerspink HJ, De Borst MH, Bakker SJL, Navis GJ. Improving the efficacy of RAAS blockade in patients with chronic kidney disease. Nat Rev Nephrol. 2013;9. https://doi.org/10.1038/ nrneph.2012.281.

10. Nishimoto M, Ohtsu H, Marumo T, Kawarazaki W, Ayuzawa N, Ueda K, et al. Mineralocorticoid receptor blockade suppressses dietary salt-induced ACEI/ARB-resistant albuminuria in nondiabetic hypertension: a sub-analysis of evaluate study. Hypertens Res. 2019. https://doi.org/10.1038/s41440-018-0201-7 (e-pub ahead of print).

11. De Borst MH, Navis G. Sodium intake, RAAS-blockade and progressive renal disease. Pharmacol Res. 2016;107:344-51.

12. Shibata S, Mu S, Kawarazaki H, Muraoka K, Ishizawa K, Yoshida $\mathrm{S}$, et al. Rac1 GTPase in rodent kidneys is essential for saltsensitive hypertension via a mineralocorticoid receptor-dependent pathway. J Clin Invest. 2011;121:3233-43.

13. Shibata S, Ishizawa K, Uchida S. Mineralocorticoid receptor as a therapeutic target in chronic kidney disease and hypertension. Hypertens Res. 2017;40:221-5.

14. Meuleman Y, Hoekstra T, Dekker FW, Navis G, Vogt L, van der Boog PJM, et al. Sodium restriction in patients with CKD: a randomized controlled trial of self-management support. Am J Kidney Dis. 2017;69:576-86.

15. Hyseni L, Elliot-Green A, Lloyd-Williams F, Kypridemos C, O'Flaherty M, McGill R, et al. Systematic review of dietary salt reduction policies: evidence for an effectiveness hierarchy? PLoS ONE. 2017;12:e177535. 